\title{
TWO NEW FINITE DIFFERENCE METHODS FOR COMPUTING EIGENVALUES OF A FOURTH ORDER LINEAR BOUNDARY VALUE PROBLEM
}

\author{
RIAZ A. USMANI \\ Department of Applied Mathematics \\ University of Manitoba \\ Winnipeg, Manitoba, Canada \\ R3T 2N2 \\ MANABU SAKAI \\ Department of Mathematics \\ University of Kagoshima \\ Kagoshima, Japan 890 \\ (Received April 5, 1985)
}

ABSTRACT. This paper describes some new finite difference methods of order 2 and 4 for computing eigenvalues of a two-point boundary value problem associated with a fourth order differential equation of the form $\left(p y^{\prime \prime}\right)^{\prime \prime}+(q-\lambda r) y=0$. Numerical results for two typical eigenvalue problems are tabulated to demonstrate practical usefulness of our methods.

KEY WORDS AND PHRASES. Band-matrices, finite-difference methods, generalized eigenvalue problem, positive definite matrices, two-point boundary value problems.

1980 AMS SUBJECT CLASSIFICATION CODE. $65 \mathrm{~L} 15$.

1. INTRODUCTION.

We shall consider the fourth order linear differential equation

$$
\frac{d^{2}}{d x^{2}}\left[p(x) \frac{d^{2} y}{d x^{2}}\right]+[q(x)-\lambda r(x)] y=0,-\infty \leq a \leq x<b<\infty,
$$

associated with the following pairs of homogeneous boundary conditions

$$
y(a)=y(b)=y^{\prime \prime}(a)=y^{\prime \prime}(b)=0 \text {. }
$$

Such boundary value problens occur in applied mathematics, engineering and modern physics, (see ref. [1-4]. In the differential equation (1.1) the functions $p(x), q(x)$, $r(x) \in C[a, b]$ and satisfy the conditions

$$
\mathrm{p}(\mathrm{x})>0, \mathrm{q}(\mathrm{x}) \geq 0 \text { and } \mathrm{r}(\mathrm{x})>0, \mathrm{x} \in[\mathrm{a}, \mathrm{b}] \text {. }
$$

We cannot compute the exact values of the eigenvalues $\lambda$ for which the boundary value problem (1.1) - (1.2) has a nontrivial eigensolution $y(x)$ for arbitrary chocies of the functions $p(x), q(x)$ and $r(x)$. We resort to numerical methods for computing approximate values of $\lambda$. The most commonly used technique for approximating $\lambda$ for which the system (1.1) - (1.2) has a nontrivial eigenfunction $y(x)$ is by finite difference methods. 
Recent1y, the author [2] has analysed some new finite different methods of order 2 and 4 for computing eigenvalues of a two point boundary value problem involving the differential equation (1.1) with $p(x) \equiv 1$ associated with one of the following pairs of homogeneous boundary conditions:

$$
\begin{aligned}
& \text { (a) } y(a)=y(b)=y^{\prime}(a)=y^{\prime}(b)=0 \\
& \text { (b) the same boundary conditions as }(1.2) \\
& \text { (c) } y(a)=y^{\prime}(a)=y^{\prime \prime}(b)=y^{\prime \prime \prime}(b)=0 \text {. }
\end{aligned}
$$

Chawla and Katti [3] have developed a numerical finite difference method of order 2 for approximating the lowest eigenvalue $\lambda$ of the system (1.1) - (1.4(a)) with $p(x)$ $\equiv 1$. A fourth order method was later developed by Chawla [4] for the numerical treatment of the same problem. This latter method leads to a generalized seven-band symmetric matrix eigenvalue problem.

Let $\lambda$ be any eigenvalue of the system (1.1) - (1.2) and let $y(x) \neq 0$ be the corresponding eigenfunction. Then on multiplying (1.1) by $y(x)$ and integrating the resulting equation from $a$ to $b$, we find after integration by parts and on using (1.2), that

$$
\lambda=\frac{\int_{a}^{b} p\left(y^{\prime \prime}\right)^{2} d x+\int_{a}^{b} q y^{2} d x}{\int_{a}^{b} r y^{2} d x}>0
$$

in view of (1.3).

The purpose of this brief report is to present two new finite difference methods for computing approximate values of $\lambda$ for the system (1.1) - (1.2). These methods lead to generalized five-band and nine-band symmetrix matrıx eigenvalue problems and provide $O\left(h^{2}\right)$ and $O\left(h^{4}\right)$-convergent approximations for the eigenvalues.

2. A SECOND ORDER METHOD

For a positive integer $N \geq 5$, let $h=(b-a) /(N+1)$ and $x_{i}=a+i h$, $i=0(1) N+1$. We shall designate $y_{i}=y\left(x_{i}\right), p_{i}=p\left(x_{i}\right), q_{i}=q\left(x_{i}\right)$ and $r_{i}=r\left(x_{i}\right)$. Note that the differential system (1.1) - (1.2) is equivalent to

$$
\begin{aligned}
& \text { (a) } y^{\prime \prime}(x)=v(x) / p(x), y(a)=y(b)=0, \\
& \text { (b) } v^{\prime \prime}(x)+[q(x)-\lambda r(x)] y(x)=0, \\
& v(a)=v(b)=0 .
\end{aligned}
$$

Now the central difference approximation to $2.1(\mathrm{a})$ is

$$
\begin{aligned}
& -y_{i-1}+2 y_{i}-y_{i+1}+h^{2}\left(v_{i} / p_{i}\right)+\frac{h^{4}}{12} y^{(4)}\left(\Theta_{i}\right)=0, \\
& \Theta_{i} \in\left(x_{i-1}, x_{i+1}\right), i=1(1) N .
\end{aligned}
$$

The preceding system can be conveniently written in matrix form

$$
J+h^{2} p^{-1} V+\frac{h^{4}}{12} T_{1}=0
$$

where $Y=\left(y_{i}\right), V=\left(v_{i}\right), T_{1}=\left(p_{i}\right)$ are $N$-dimensional column vectors with $\rho_{i}=y^{(4)}\left(\theta_{i}\right), P=\operatorname{diag}\left(p_{i}\right)$, and $J=\left(j_{m n}\right)$ is a tridiagonal matrix so that 


$$
j_{m} n=\left\{\begin{aligned}
2, & m=n \\
-1, & |m-n|=1 \\
0, & |m-n|>1 .
\end{aligned}\right.
$$

In an analogous manner, on discretizing $2.1(\mathrm{~b})$, we get

$$
J V-h^{2} Q Y+\lambda h^{2} R Y+\frac{h^{4}}{12} T_{2}=0
$$

where $Q=\operatorname{diag}\left(q_{i}\right), R=\operatorname{diag}\left(r_{i}\right)$ and $T_{2}=\left(\sigma_{i}\right)$ with $\sigma_{i}=v^{(4)}\left(\phi_{i}\right)$, $\phi_{i} \in\left(x_{i-1}, x_{i+1}\right)$. Next, we eliminate $v$ between $(2.3)$ and $(2.5)$ to obtain

$$
A Y \equiv\left(J P J+h^{4} Q\right) Y=\lambda h^{4} R Y+\Gamma,
$$

where

$$
\Gamma=\frac{1}{12}\left[h^{6} T_{2}-h^{4} J P T_{1}\right]
$$

It can be verified that the matrix $A=J P J+h^{4} Q$ is a five-band symmetric matrix. Now, in (2.6), neglect truncation error $\Gamma$, replace $Y$ by $\tilde{Y}$, then our method for computing approximations $\wedge$ for $\lambda$ of the system (1.1) - (1.2) can be expressed as a generalized seven-band symmetric matrix eigenvalue problem

$$
A \tilde{Y}=\Lambda h^{4} R \tilde{Y} \text {. }
$$

In fact the matrix JPJ is a positive definite matrix and hence for any step-size $\mathrm{h}>0$, the approximations $\wedge$ for $\lambda$ by (2.8) are real and positive for all $\mathrm{p}(\mathrm{x})>0$ and $r(x)>0$. That our method provides $0\left(h^{2}\right)$ convergent approximations $\wedge$ for $\lambda$ can be established following Grigorieff [5]. We omit the proof of convergence for brevity.

3. A FOURTH ORDER METHOD

Following Shoosmith [6] the boundary value problems $2.1(a)$ and $2.1(b)$ are discretized by the finite difference scheme

$$
\begin{aligned}
& \text { (a) } 14 y_{0}-29 y_{1}+16 y_{2}-y_{3}=h^{2}\left[y_{0}^{\prime \prime}+12 y_{1}^{\prime \prime}\right], \\
& \text { (b) }\left(1-\frac{\delta^{2}}{12}\right) \delta^{2} y_{i}=h^{2} y_{i}^{\prime \prime}, i=2(1) N-1, \\
& \text { (c) }-y_{n-2}+16 y_{N-1}-29 y_{N}+14 y_{N+1}=h^{2}\left[12 y_{N}^{\prime \prime}+y_{N+1}^{\prime \prime}\right] .
\end{aligned}
$$

It turns out the boundary value problem 3.1 (a) gives rise to the linear equations

$$
\tilde{M Y}+12 h^{2} p^{-1} \tilde{V}=0
$$

Similarly, for the system $2.1(\mathrm{~b})$, we obtain the linear equations

$$
M \tilde{V}=12 h^{2} Q \tilde{Y}-12 \Lambda h^{2} R \tilde{Y},
$$


where the five-band $\mathrm{N} \times \mathrm{N}$ matrix $\mathrm{M}$ is given by

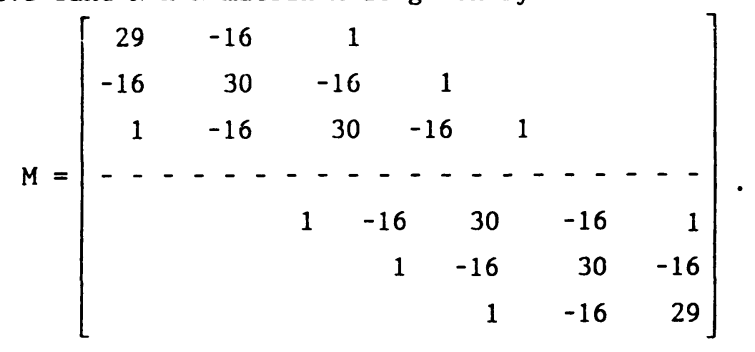

The elimination of $\tilde{\mathrm{V}}$ from (3.2) and (3.3) gives our method for computing $\Lambda$ for $\lambda$ of (1.1) - (1.2) in the form

$$
\left(M P M+144 h^{4} Q\right) \tilde{Y}=144 \Lambda h^{4} R \tilde{Y} \text {, }
$$

where the matrix MPM is a nine-band positive definite matrix and hence for any step-size $h>0$, the approximations $\Lambda$ for $\lambda$ by (3.5) are real and positive for all $p(x), r(x)>0$. As before, it can be proved from the results of Grigorieff [5] that our present method provided $0\left(h^{4}\right)$ convergent approximations $\Lambda$ for $\lambda$.

4. NUMERICAL RESULTS

In order to illustrate our methods of order 2 and 4 for the approximation of $\lambda$ satisfying $(1.1)-(1.2)$, we consider the eigenvalue problems:

$$
\begin{aligned}
& {\left[\left(1+x^{2}\right) y^{\prime \prime}\right]^{\prime \prime}+\left[\frac{1}{\left(1+x^{2}\right)}-\lambda(1+x)^{4}\right] y=0,} \\
& y(0)=y(1)=y^{\prime \prime}(0)=y^{\prime \prime}(1)=0 .
\end{aligned}
$$

The smallest eigenvalue $\lambda_{1}=22.754,058,480, .$.

$$
\begin{aligned}
& {\left[e^{x} y^{\prime \prime}\right] "+[\sin x-\lambda \cos x] y=0} \\
& y(0)=y(1)=y^{\prime \prime}(0)=y^{\prime \prime}(1)=0
\end{aligned}
$$

The smallest eigenvalue of the system $(4.2)$ is $\lambda_{1}=181.345,488,233,$. . We list the approximations $\Lambda_{1}$ for $\lambda_{1}$ and the relative errors $\left|1-\frac{\lambda_{1}}{\Lambda_{1}}\right|$ for various values of the step-size $h$. It is readily verified that the relative errors (Table I) based on generalized eigenvalue problem $(2.8)$ provide $0\left(h^{2}\right)$ - convergent approximations for the smallest eigenvalue of the system (4.1) and (4.2). Similarly, the relative errors (Table II) based on the generalized eigenvalue problem (3.5) do indeed provide $0\left(h^{4}\right)$-convergent approximations for the smallest eigenvalue of the systems (4.1) and (4.2). 
TABLE I

Results based on (2.8), second order approximations

\begin{tabular}{lrrl}
\hline Problem & $\mathrm{N}$ & $\Lambda_{1}$ & $\left|1-\frac{\lambda_{1}}{\Lambda_{1}}\right|$ \\
\hline$(4.1)$ & 7 & 22.187 & $2.557-2 *$ \\
& 15 & 22.610 & $6.352-3$ \\
& 31 & 22.718 & $1.586-3$ \\
& 63 & 22.745 & $3.962-4$ \\
& 127 & 22.752 & $9.907-5$ \\
& 255 & 22.753 & $2.480-5$ \\
\hline$(4.2)$ & 7 & 176.641 & $2.664-2$ \\
& 15 & 180.159 & $6.588-3$ \\
& 31 & 181.048 & $1.642-3$ \\
& 63 & 181.271 & $4.103-4$ \\
& 127 & 181.327 & $2.560-5$ \\
& 255 & 181.341 &
\end{tabular}

*We write $2.557-2$ for $2.557 \times 10^{-2}$.

TABLE II

Results based on (3.5), 4th order approximations

\begin{tabular}{ccc|c}
\hline Problem & $N$ & $\Lambda_{1}$ & $\left|1-\frac{\lambda^{1}}{\Lambda_{1}}\right|$ \\
\hline$(4.1)$ & 7 & $22.746,419$ & $3.358-4$ \\
& 15 & $22.753,574$ & $2.129-5$ \\
& 31 & $22.754,027$ & $1.358-6$ \\
& 63 & $22.754,056$ & $1.078-7$ \\
\hline$(4.2)$ & 7 & $181.244,637$ & $5.564-4$ \\
& 15 & $181.339,089$ & $3.529-5$ \\
& 31 & $181.345,093$ & $2.175-6$ \\
& 63 & $181.345,470$ & $9.728-8$ \\
\hline
\end{tabular}

ACKNOWLEDGEMENT. This work was supported in part by a grant from the Natural Sciences and Engineering Research Council of Canada. The author also acknowledges the assistance of Mr. Manzoor Hussain for making numerical calculations presented in Tables I and II.

\section{REFERENCES}

1. HILDEBRAND, F.B. Advanced Calculus for Applications, Prentice-Hall, Inc., Englewood Cliffs, N.J., 1964.

2. USMANI, R. A. Finite-Difference Methods for Computing Eigenvalues of Fourth Order Boundary Value Problems, International J. of Mathematics and Mathematical Sciences, 9 (1986), 137-143.

3. CHAWLA, M.M. and KATTI, C.P. A New Symmetric Five-Diagonal Finite Difference Method for Computing Eigenvalues of Fourth Order Two-Point Boundary Value Problems, J. Computional and Applied Mathematics, $\underline{8}$ (1982) 135-136.

4. CHAWLA, M.M. A New Fourth-Order Finite Difference Method for Computing Eigenvalues of Fourth-Order Two-Point Boundary Value Problem, IMA Journal of Numerical Analysis, $\underline{3}$ (1983) 291-293.

5. GRIGORIEFF, R.D. Discrete Approximation Von Eigenwertproblemen II, Nunerische Mathematik, 24(1975) 415-433.

6. SHOOSMITH, J.N. A Study of Monotone Matrices with Application to the High-Order, Finite-Difference Solution of a Linear, Two-Point Boundary Value Problem, Ph.D. Dissertation, August 1973, University of Virginia. 


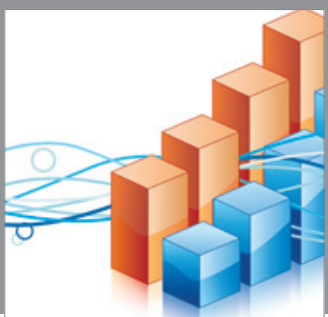

Advances in

Operations Research

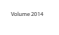

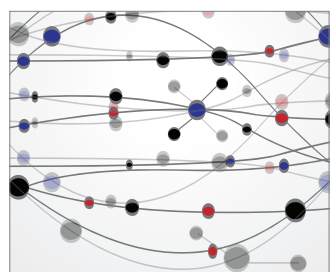

\section{The Scientific} World Journal
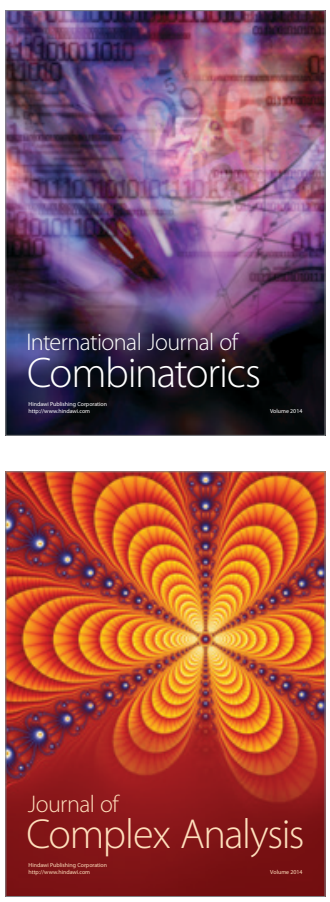

International Journal of

Mathematics and

Mathematical

Sciences
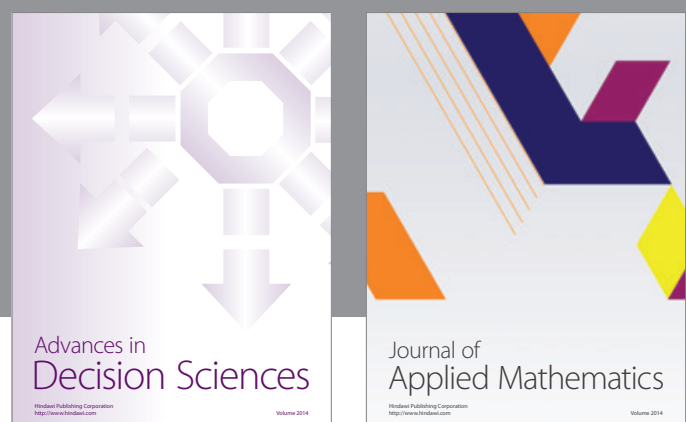

Journal of

Applied Mathematics
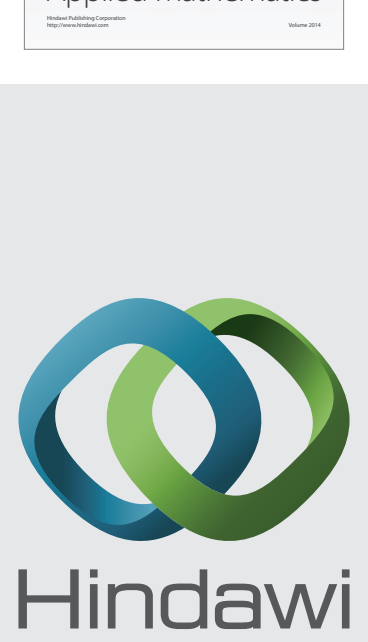

Submit your manuscripts at http://www.hindawi.com
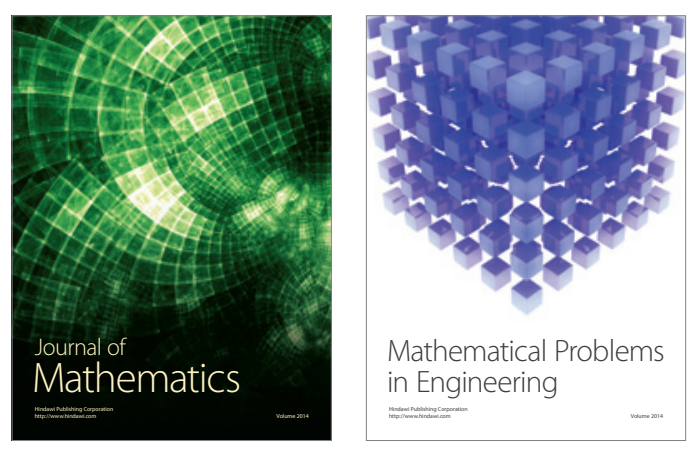

Mathematical Problems in Engineering
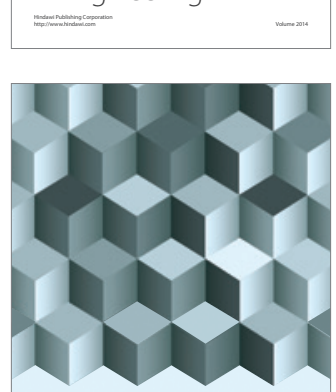

Journal of

Function Spaces
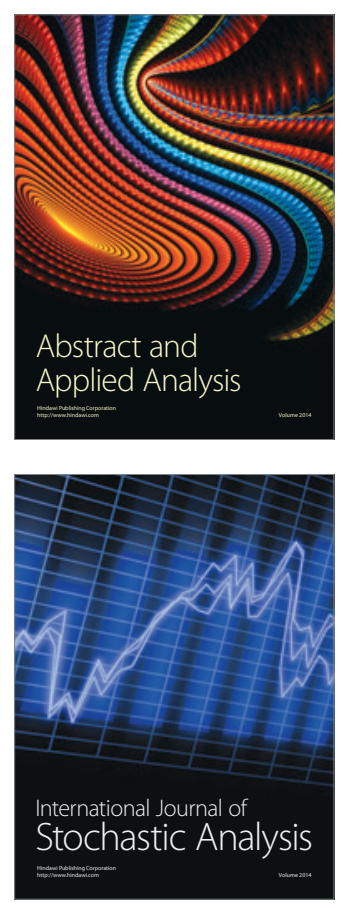

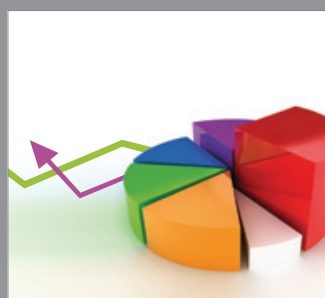

ournal of

Probability and Statistics

Promensencen
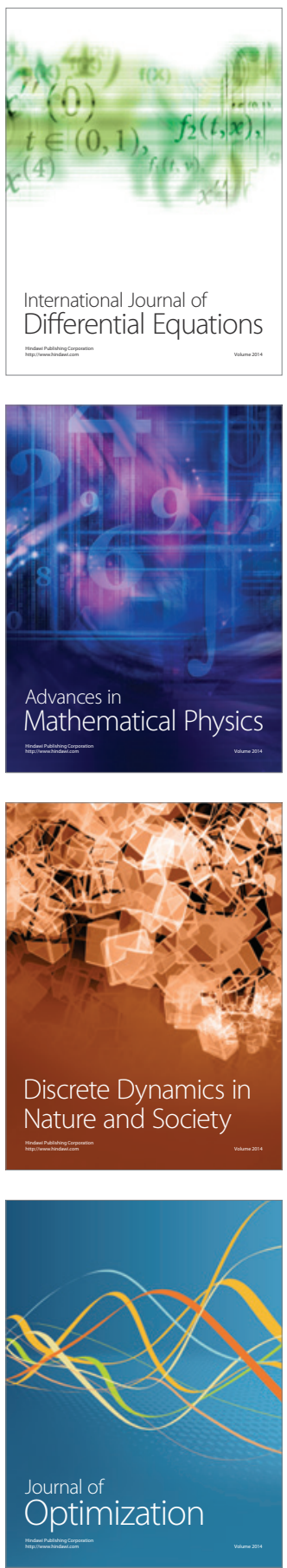